\title{
Some properties of equivalent fuzzy norms
}

\author{
Gil Seob Rhie and In Ah Hwang \\ Department of Mathematics, Hannam University \\ Daejeon 306-791 Korea.
}

\begin{abstract}
In the present paper, , we observe a relation between fuzzy norms and induced crisp norms on a linear space. We first prove that if $\rho_{1}, \rho_{2}$ are equivalent fuzzy norms on a linear space, then for every $\varepsilon \in(0,1)$, the induced crisp norms $P_{\varepsilon}^{1}$ and $P_{\varepsilon}^{2}$ respectively are equivalent. Since the converse does not hold, we prove it under some strict conditions. And consider the following theorem proved in [8]: Let $\rho$ be a lower semicontinuous fuzzy norm on a normed linear space $X$, and have the bounded support. Then $\rho$ is equivalent to the fuzzy norm $\chi_{B}$ where $B$ is the closed unit ball of $X$. The lower semi-continuity of $\rho$ is an essential condition which guarantees the continuity of $P_{\varepsilon}$ where $0<\varepsilon<1$. As the last result, we prove that : if $\rho$ is a fuzzy norm on a finite dimensional vector space, then $\rho$ is equivalent to $\chi_{B}$ if and only if the support of $\rho$ is bounded.
\end{abstract}

Key words : fuzzy normed linear space, equivalent fuzzy norm, induced crisp norm.

\section{Introduction}

Katsaras and Liu[3] introduced the notion of a fuzzy vector space and fuzzy topological vector space. These ideas were modified by Katsaras[1] and in [2] he defined a fuzzy norm on a vector space. Krishna and Sarma [4] studied the topological generation and normability of fuzzy topological vector spaces by observing the equivalence of fuzzy topology on a vector space, obtained in several different ways[4]. They also give a sufficient condition for the fuzzy vector topology induced by a family of fuzzy seminorms to be fuzzy normable (i.e.) to be induced by a single fuzzy norm. One interesting observation made in [4] was that if $\rho$ is a fuzzy (semi) norm on a linear space $X$, then for each $\varepsilon$ with $0<\varepsilon<1$, $P_{\varepsilon}(x)=\inf \{t>0 \mid t \rho(x)>\varepsilon\}$ gives an ordinary (semi)norm on $X$. This idea was used to obtain the equivalence of several fuzzy topologies mentioned earlier.

In the present paper, we observe a relation between fuzzy norms and induced crisp norms on a linear space. We first prove the following theorem: Let $\rho_{1}, \rho_{2}$ be two fuzzy norms on a linear space and $P_{\varepsilon}^{1}, P_{\varepsilon}^{2}$ be induced crisp norms respectively. If $\rho_{1}, \rho_{2}$ are equivalent, then for every $\varepsilon \in(0,1)$, the induced crisp norms $P_{\varepsilon}^{1}$ and $P_{\varepsilon}^{2}$ are equivalent. Since the converse is not true, we prove the converse under some strict conditions. And the following theorem was proved in [8]: Let $\rho$ be a lower semicontinuous fuzzy norm on a normed linear space $X$ and have the bounded support : $x \in X \mid \rho(x)>0$ is bounded. Then $\rho$ is equivalent to the fuzzy norm $\chi_{B}$ where $B$ is the closed unit

Manuscript received May. 24, 2005; revised Jun. 8, 2005. This work was supported by Hannam University Grant in 2005 ball of $X$. The lower semicontinuity of $\rho$, in the hypothsis of the theorem, is a very important condition which guarantees the continuity of $P_{\varepsilon}$ where $0<\varepsilon<1$. Removing the lower simicontinuity of $\rho$, as the last result, we prove that if $\rho$ is a fuzzy norm on a finite dimensional vector space, then $\rho$ is equivalent to $\chi_{B}$ where $B$ is the closed unit ball of $X$ if and only if the support of $\rho$ is bounded.

\section{Preliminaries}

Let $X$ be a linear space over the field $K(R$ or $C)$ throughout this paper. A fuzzy set in $X$ is an element of the set $I^{X}$ of all functions from $\mathrm{X}$ into the unit interval $I$. In general, fuzzy subsets of $\mathrm{X}$ are denoted by Greek letters. $\chi_{A}$ denotes the characteristic function of the set $A$. $\vee$ and $\wedge$ are used for the supremum and infimum of a family, respectively, $R_{+}=[0, \infty)$.

Definition 2.1[3]. If $\mu \in I^{X}$ and $t \in K$, then

$$
(t \mu)(x)=\left\{\begin{array}{lll}
\mu(x / t) & \text { if } & t \neq 0 \\
0 & \text { if } & t=0 \text { and } x \neq 0 \\
\vee_{y \in X} \mu(y) & \text { if } & t=0 \text { and } x=0
\end{array}\right.
$$

Definition 2.2[3]. $\mu \in I^{X}$ is said to be

(a) convex if $t \mu+(1-t) \mu \subseteq \mu$ for each $t \in[0,1]$,

(b) balanced if $t \mu \subseteq \mu$ for each $t \in K$ with $|t| \leq 1$,

(c) absolutely convex if $\mu$ is convex and balanced,

(d) absorbing if $\vee_{t>0} t \mu(x)=1$ for all $x \in X$.

Definition 2.3[2]. A fuzzy seminorm $\rho$ on $\mathrm{X}$ is a fuzzy 
subset of $\mathrm{X}$ which satisfies the following three conditions.

(a) $\rho$ is convex,

(b) $\rho$ is balanced,

(c) $\rho$ is absorbing.

If, in addition, a fuzzy seminorm $\rho$ satisfies the condition

(d) $\wedge_{t>0} t \rho(x)=0$ for $x \neq 0$ in $\mathrm{X}$, then $\rho$ is called a fuzzy norm.

Theorem 2.4[2]. If $\rho$ is a fuzzy seminorm on $X$, then the family

$B=B_{\rho}=\{\theta \wedge(t \rho): t>0,0<\theta \leq 1\}$

is a base at zero for a fuzzy linear topology $\tau_{\rho}$.

Definition 2.5.[2]. A linear space equipped with a fuzzy seminorm (resp. fuzzy norm) is called a fuzzy seminormed (resp. fuzzy normed) linear space.

Definition 2.6[2]. Two fuzzy seminorms $\rho_{1}, \rho_{2}$ on a vector space $X$, are said to be equivalent if $\tau_{\rho_{1}}=\tau_{\rho_{2}}$.

Theorem 2.7[2]. The fuzzy seminorms $\rho_{1}, \rho_{2}$ on a vector space $X$ are equivalent iff the following condition is satisfied : For each $0<\theta<1$ there exists $t>0$ such that $\theta \wedge \rho_{1}(t x) \leq \rho_{2}(x)$ and $\theta \wedge \rho_{2}(t x) \leq \rho_{1}(x)$.

Theorem 2.8[4]. Let $\rho$ be a fuzzy seminorm on a linear space $X$. For each $\varepsilon \in(0,1)$, difine

$P_{\varepsilon}(x)=\wedge\{t>0 \mid t \rho(x)>\varepsilon\}$.

Then $P_{\varepsilon}$ is a seminorm on $X$ for each $\varepsilon \in(0,1)$. Further $\rho$ is a fuzzy norm on $X$ if and only if for each $\varepsilon \in(0,1), P_{\epsilon}$ is a norm on $X$.

Remark (a)The above $P_{\varepsilon}: X \rightarrow R_{+}$is the Minkowski functional of the convex balanced absorbing set $A_{\varepsilon}$ $=\{x \in X|\rho(x)\rangle \varepsilon\}$.

(b) The function $P: X \rightarrow R_{+}$defined by $P(x)=\wedge\left\{P_{\varepsilon}(x) \mid \varepsilon \in(0,1)\right\}$ is a seminorm on $\mathrm{X}$. The proof is clear.

\section{Main results}

In this section, we observe a relation between the equivalence of fuzzy norms and that of induced crisp norms on a linear space. We begin with the following theorem.

Theorem 3.1 Let $\rho_{1}, \rho_{2}$ be two fuzzy norms on a vector space $X$ and $\theta \in(0,1)$. If $\theta \wedge \rho_{1}(x) \leq \rho_{2}(x)$ for all $x \in X$, then for every $\varepsilon \in(0, \theta), P_{\varepsilon}^{1}(x) \geq P_{\varepsilon}^{2}(x)$ for all $x \in X$.

proof. Since $\theta \wedge \rho_{1}(x) \leq \rho_{2}(x)$ for all $x \in X$, for every $t>0, \quad \theta \wedge t \rho_{1}(x) \leq t \rho_{2}(x)$. Let $\varepsilon \in(0, \theta)$ and $x \in X$ be fixed. Since $\theta \wedge t \rho_{1}(x)>\varepsilon$ implies $t \rho_{2}(x)>\varepsilon$, $\left\{t>0 \mid \theta \wedge t \rho_{1}(x)>\varepsilon\right\}$ is a subset of $\left\{t^{\prime}>0 \mid t^{\prime} \rho_{2}(x)>\varepsilon\right\}$. Whence

$$
\begin{aligned}
P_{\varepsilon}^{\mathrm{l}}(x) & =\inf \left\{t>0 \mid t \rho_{1}(x)>\varepsilon\right\} \\
& =\inf \left\{t>0 \mid \theta \wedge t \rho_{1}(x)>\varepsilon\right\} \\
& \geq \inf \left\{t>0 \mid t \rho_{2}(x)>\varepsilon\right\} \\
& =P_{\varepsilon}^{2}(x) .
\end{aligned}
$$

This completes the proof.

Theorem 3.2 Let $\rho_{1}$ and $\rho_{2}$ be two equivalent fuzzy norms on a linear space $X$. then for every $\varepsilon \in(0,1)$, the induced crisp norms $P_{\epsilon}^{1}$ and $P_{\varepsilon}^{2}$ are equivalent.

proof. Let $\varepsilon \in(0,1)$ and $\theta \in(\varepsilon, 1)$. Then there exists a positive real number $s=s(\theta)>1$ such that $\theta \wedge \rho_{1}(s x) \leq \rho_{2}(x)$ and $\theta \wedge \rho_{2}(s x) \leq \rho_{1}(x)$ for all $x \in X$. By the preceding theorem, $P_{\varepsilon}^{2}(x) \leq s P_{\varepsilon}^{1}(x)$ for all $x \in X$ and $P^{1}{ }_{\varepsilon}(x) \leq s P_{\varepsilon}^{2}(x) \quad$ for $\quad$ all $\quad x \in X$. And so $\frac{1}{s} P_{\varepsilon}^{2}(x) \leq P_{\varepsilon}^{1}(x) \leq s P_{\varepsilon}^{2}(x)$ for all $x \in X$. That is, two induced crisp norms $P_{\epsilon}^{1}$ and $P_{\epsilon}^{2}$ are equivalent for every $\varepsilon \in(0,1)$. This completes the proof.

Remark. Consider the following two fuzzy norms on $R^{2}$. Let $\rho_{1}$ and $\rho_{2}$ be defined as $\rho_{1}(x, y)=\chi_{B}(x, y)$ where $\mathrm{B}$ is the closed unit ball of $R^{2}$

$$
\text { and } \quad \rho_{2}(x, y)=\frac{1}{1+\left(x^{2}+y^{2}\right)} \text {. }
$$

Then it is clear that two fuzzy norms $\rho_{1}, \rho_{2}$ are not equivalent. Since every two norms defined on a finite dimensional vector space are equivalent, the converse of the preceding theorem is not true. By reason of the above description, we prove the converse of the theorem under sufficiently restricted conditions. We begin with the following Lemma.

Lemma 3.3 Let $\rho$ be a fuzzy seminorm on a linear space $X$ and $\rho(x)=\inf \{\rho(t x) \mid 0<t<1\}$ for all $x \in X$. If $x_{0} \in X$ and $\rho\left(x_{0}\right)<\varepsilon<1$, then $P_{\varepsilon}\left(x_{0}\right)>1$.

proof. Since $P_{\varepsilon}\left(x_{0}\right)=\wedge\left\{t>0 \mid t \rho\left(x_{0}\right)>\varepsilon\right\} \geq 1$, we will show that $P_{\varepsilon}\left(x_{0}\right) \neq 1$. For this, let $P_{\epsilon}\left(x_{0}\right)=1$. Then $t \rho\left(x_{0}\right)>\varepsilon$ for all $t>1$. Since $\rho(x)=\inf \{\rho(t x) \mid 0<t<1\}$ for all $x \in X$,

$$
\begin{aligned}
\rho\left(x_{0}\right) & =\wedge\left\{\rho\left(s x_{0}\right) \mid 0<s<1\right\} \\
& =\wedge\left\{t \rho\left(x_{0}\right) \mid t>1\right\} \\
& \geq \varepsilon
\end{aligned}
$$

which contradicts the fact $\varepsilon>\rho\left(x_{0}\right)$. Therefore $P_{\varepsilon}\left(x_{0}\right)>1$. This completes the proof. 
Following example shows that the condition that $\rho(x)=\inf \{\rho(t x) \mid 0<t<1\}$ for all $x \in X$, in the hypothesis of the preceding lemma, is essential. Let a fuzzy norm $\rho$ is given on a real line by

$$
\rho(x)=\left\{\begin{array}{ccc}
1 & \text { on } & (-1,1) \\
\frac{1}{2} & \text { on } & {[-2,-1] \cup[1,2]} \\
0 & & \text { otherwise }
\end{array}\right.
$$

and $\varepsilon=\frac{2}{3}$. Then $\rho(1)<\varepsilon$ but $P_{\varepsilon}(1)=1$.

Theorem 3.4 Let $\rho_{1}, \rho_{2}$ be two fuzzy seminorms on a linear space $\mathrm{X}$ and for every $x$ in $\mathrm{X}$, $\rho_{2}(x)=\inf \left\{\rho_{2}(t x) \mid 0<t<1\right\}$. If for every $\varepsilon \in(0, \theta)$ $P_{\varepsilon}^{1}(x) \geq P_{\varepsilon}^{2}(x)$ for all $x \in X$, then for every $x \in X$, $\theta \wedge \rho_{1}(x) \leq \rho_{2}(x)$.

proof. Suppose that there exists $y \in X$ such that $\rho_{2}(y)<\theta \wedge \rho_{1}(y)$. Let $\rho_{2}(y)<\varepsilon<\theta \wedge \rho_{1}(y)$ be given. Then

$$
\begin{aligned}
P_{\varepsilon}^{1}(y) & =\inf \left\{t>0 \mid t \rho_{1}(y)>\varepsilon\right\} \\
& =\inf \left\{t>0 \mid \theta \wedge t \rho_{1}(y)>\varepsilon\right\} \leq 1 .
\end{aligned}
$$

Since $P_{\varepsilon}^{2}(y)>1$ by above Lemma,$P_{\varepsilon}^{2}(y)>P_{\varepsilon}^{1}(y)$ which contradicts that for every $\varepsilon \in(0, \theta), P_{\varepsilon}^{2}(x) \leq P_{\varepsilon}^{1}(x)$ for all $x \in X$. Therefore $\theta \wedge \rho_{1}(x) \leq \rho_{2}(x)$ for all $x \in X$.This completes the proof.

Theorem 3.5 Let $\rho_{1}, \rho_{2}$ be fuzzy norms and for every $i=1,2 \rho_{i}(x)=\inf \left\{\rho_{i}(t x) \mid 0<t<1\right\}$ for all $x \in X$. If there exists $m, M>0$ such that for every $\varepsilon \in(0,1)$, $m P_{\varepsilon}^{1}(x) \leq P_{\varepsilon}^{2}(x) \leq M P_{\varepsilon}^{1}(x)$ for all $x \in X$, then $\rho_{1}$ and $\rho_{2}$ are equivalent.

proof. Let $\theta \in(0,1)$. Since for every $\varepsilon \in(0, \theta)$, $m P_{\varepsilon}^{1}(x) \leq P_{\varepsilon}^{2}(x)$ and $P_{\varepsilon}^{2}(x) \leq M P_{\varepsilon}^{1}(x)$ for all $x \in X$, by the above theorem, we get

$$
\begin{aligned}
& \theta \wedge \rho_{2}\left(\frac{1}{m} x\right) \leq \rho_{1}(x) \text { for all } x \in X \text { and } \\
& \theta \wedge \rho_{1}(M x) \leq \rho_{2}(x) \text { for all } x \in X .
\end{aligned}
$$

Take $t=\max \left\{\frac{1}{m}, M\right\}$, then $\theta \wedge \rho_{2}(t x) \leq \rho_{1}(x)$ and $\theta \wedge \rho_{1}(t x) \leq \rho_{2}(x)$ for all $x \in X$.

This completes the proof.

Proposition 3.6 [7]. Let $(V, T)$ be a topological linear space over $K$, And let $B \subset V$ be a convex balanced absorbing set, and let $\mathrm{q}$ be the Minkowski functional of $\mathrm{B}$. Then $q$ is a seminorm and

(a) $q: V \rightarrow R$ is continuous if and only if $0 \in \operatorname{int}(B)$.

(b) If $B$ is bounded, then $\mathrm{q}$ is a norm.

Theorem 3.7 [8] Let $\rho$ be a lower semicontinuous fuzzy norm on a normed linear space $X$, and have the bounded support: $\{x \in X \mid \rho(x)>0\}$ is bounded. Then $\rho$ is equivalent to the fuzzy norm $\chi_{B}$ where $B$ is the closed unit ball of $X$.

By 'Theorem 3.2 and Proposition 3.6 and Theorem 3.7, we obtain the following theorem.

Theorem 3.8 Let $(X,\|\|$.$) be a normed linear space.$ Let $\rho$ be a lower semicontinuous fuzzy norm on $X$ and have the bounded support. Then every $P_{\varepsilon}$ with $0<\varepsilon<1$ is equivalent to the norm $\|$.$\| .$

The lower semicontinuity of $\rho$, in the hypothsis of Theorem 3.7, is an essential condition which guarantees the continuity of $P_{\varepsilon}$ where $0<\varepsilon<1$. Removing the lower simicontinuity of $\rho$, in the finite dimensional case, we prove the next theorem which is our last result.

Theorem 3.9. Let $\rho$ be a fuzzy norm on a finite dimensional vector space $(X,\|\|$.$) . Then \rho$ is equivalent to $\chi_{B}$ where $B$ is the closed unit ball of $X$ if and only if the support of $\rho$ is bounded.

Proof. Suppose that $\rho$ has the bounded support. Let $\theta \in(0,1)$ be given. Since $\rho$ is a fuzzy norm in $X$, the set $A_{\theta}=\{x \in X \mid \rho(x)>\theta\}$ is convex balanced and absorbing, and $P_{\theta}$ is the Minkowski functional of $A_{\theta}$. Since every two norms on a finite dimensional vector space are equivalent and $P_{\theta}:\left(X, P_{\theta}\right) \rightarrow K$ is continuous, $P_{\theta}:(X,\|\|.) \rightarrow K$ is continuous.

By Proposition 3.6, zero vector 0 is an interior point of $A_{\theta}$. Thus there exists $t_{1}>0$ such that $t_{1}^{-1} B \subset A_{\theta}$. Hence for every $x \in X, \theta \wedge \chi_{B}\left(t_{1} x\right)=\theta \times \chi_{B}\left(t_{1}{ }^{\prime} x\right) \leq \rho(x)$.

Since $\rho$ has the bounded support, there exists $t_{2}>0$ such that $\quad$ suppo $\subset t_{2} B$ and so $\|x\|>t_{2}$ implies $\rho(x)=0$. Therefore $\rho\left(t_{2} x\right) \leq \chi_{B}(x)$ for all $x \in X$. Take $t_{\theta}=t_{1} \vee t_{2}$. Then $\theta \wedge \chi_{B}\left(t_{\theta} x\right) \leq \rho(x)$ and $\theta \wedge \rho\left(t_{\theta} x\right) \leq \chi_{B}(x)$ for all $x \in X$. This proves that $\rho$ and $\chi_{B}$ are equivalent.

For the opposite direction, let $\rho$ and $\chi_{B}$ be equivalent.

Let a $\theta \in(0,1)$ be given. Then there exists $t>0$ such that $\theta \wedge \rho(t x) \leq \chi_{B}(x)$ for all $x \in X$. From this fact, we get that if $0<\rho(x)$ then $\chi_{t B}(x)>0$, Equivalently the support of $\rho$ is a subset of $t B$. Hence $\rho$ has the bounded support. This completes the proof.

\section{References}

[1] A.K. Katsaras, Fuzzy topological vector space I, Fuzzy Sets and Systems 6 (1981) 85-95.

[2] A.K. Katsaras, Fuzzy topological vector space П, Fuzzy Sets and Systems 12 (1984) 143-154.

[3] A.K. Katsaras and D.B. Liu, Fuzzy vector spaces and 
International Journal of Fuzzy Logic and Intelligent Systems, vol. 5, no. 2, June 2005

fuzzy topolpgical vector spaces, J. Math. Anal. Appl. 58 ( 1977) 135-146.

[4] S.V. Krishna and K.K.M. Sarma, Fuzzy topological vector spaces - topolpgical generation and normability, Fuzzy Sets and Systems 41 (1991) 89-99.

[5] S.V.Krishna and K.K.M. Sarma, Fuzzy continuity of linear maps on vector spaces, Fuzzy Sets and systems 45 (1992) 341-354.

[6] S.V.Krishna and K.K.M. Sarma, Separation of fuzzy normed linear spaces, Fuzzy Sets and Systems 63 (1994) 207-217.

[7] R. Larsen, Functional Analysis, Marcel Dekker, New York, 1973.

[8] G.S. Rhie, B.M Choi and D.S. Kim, On the completeness of fuzzy normed linear spaces, Math. Japonica 45 (1) (1997) 33-37.

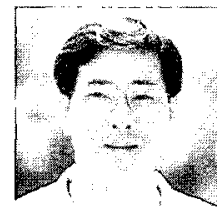

Gil Seob Rhie

$\mathrm{He}$ is currently a professor in Hannam University, Taejon, Korea. His main research interests include fuzzy analysis and analysis.

Phone : $+82-42-629-7453$

Fax : $+82-42-629-7894$

E-mail : gsrhie@hanmail.net

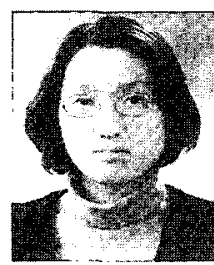

\section{In Ah Hwang}

She is currently a lecturer in Hannam University, Taejon, Korea Her main research interests include fuzzy analysis and fuzzy topology.

Phone : +82-42-629-7453

Fax : $+82-42-629-7894$ 